\title{
Evaluation of Severity of Cardiac Ischemia Using In Silico ECG Computed From 2D Reaction Diffusion Model
}

\author{
S E Loeffler and J M Starobin \\ University of North Carolina at Greensboro, Greensboro NC, USA
}

\begin{abstract}
This study focuses on the analysis of the bioelectrical activity of the left ventricle using a 2D Bueno-OrovioFenton-Cherry monodomain reaction diffusion model. ECGs signals are simulated for normal and ischemic conditions of varying severity. Changes in ischemia are examined in a single precordial lead as the size of the ischemic area increases in various locations. Analyzing this single lead ECG, we determine the ratio between $S T$ deviation and T-wave amplitude and establish a threshold sufficient for monitoring acute ischemic event. This method may be potentially implemented to predict sudden cardiac death.
\end{abstract}

\section{Introduction}

Among all causes of mortality cardiovascular disease (CVD) ranks near the top representing $31 \%$ of all global deaths and amounts to 17.9 million people who died from CVD in 2016 [1]. The CVD, which is an occlusion of coronary arteries, often results in a deficiency of oxygen supply known as cardiac ischemia. Acute form of this disorder poses a significant danger since it may frequently result in heart attack and cardiac death.

A common approach to predict the development of cardiac ischemia is tracking a body surface electrocardiogram (ECG). Using the forward problem of electrocardiology [2], a body surface ECG may be simulated utilizing reaction diffusion (RD) equations which describe ionic processes inside a myocardium. The reaction part of these equations characterizes the flow of ions through cellular membrane channels. The diffusion part describes the diffusive flow of charges between the neighboring cellular membranes. Cumulatively, these processes determine the dynamics of cellular transmembrane potentials (action potential, AP) and ascertain the magnitude of electric field of the heart induced at the surface of the body.

Our approach is centered on numerical simulations of body surface ECGs signals by using the RD models with simplified geometries. Though detailed 3D models are commonly preferred[3], the simplified 2D models have also been proved to provide valuable information by keeping intact fundamental features of excitation in normal and ischemic myocardium[4].

It is well known that myocardial ischemia raises cellular membrane resting potential, shortened AP duration and decreases the AP upstroke velocities [5], [6]. In turn, these changes affect the surface ECG by shifting its baseline voltage and altering the morphology of the ST segment, QRS complex and T-wave. We will study in silico ECG signals computed from the $2 \mathrm{D}$ reactiondiffusion model and will evaluate the severity of cardiac ischemia for various model parameters.

\section{Methods}

\subsection{Model Geometry}

For the average adult male, the distance from the center of the base of the Left Ventricle (LV) to its inner wall is $\sim 2 \mathrm{~cm}$. It is shorter than the distance from the LV base to the inner wall of the apex $\sim 6 \mathrm{~cm}$. Generally, the myocardial thickness varies from $\sim 1 \mathrm{~cm}$ at the base to $\sim 2$ $\mathrm{cm}$ at the apex. Using these dimensions, the coronal cross section of the LV may be approximated as a 2D symmetric horseshoe (Fig. 2). The horseshoe is made of two concentric ellipses with the same axes. The inner and outer ellipses have semi-major and semi-minor axis of 6 and $2 \mathrm{~cm}$ and 8 and $3 \mathrm{~cm}$, respectively. Using these proportions, the following coordinate system can be utilized to approximate the coronal cross section of $\mathrm{LV}$ (Eq. 1)

$$
a=\sqrt{x^{2}+\frac{y^{2}}{s^{2}}} \quad \theta=\arctan \left(\frac{y}{s x}\right)
$$

Here, $x$ and $y$ are the Cartesian coordinates and $s$ is the scaling value to fit two concentric ellipses with different semi-major and semi-minor axes lengths. Parameter $s$ is the slope of the regression line " $y=\mathrm{s} x$ ", which is equal to $2.796\left(R^{2}=.84\right)$. Such fitting corresponds to semiminor axis of 2 and $3 \mathrm{~cm}$ and to semi-major axis of 5.54 and $8.30 \mathrm{~cm}$, respectively (Fig. 2).

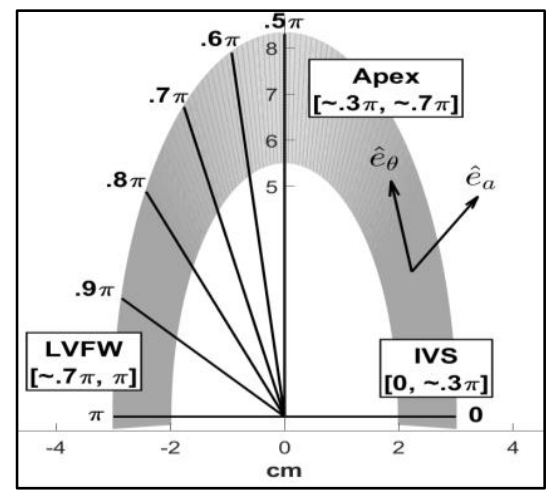


Figure 1. Horseshoe model geometry in a non-orthogonal basis $\left(\hat{e}_{a}, \hat{e}_{\theta}\right)$. Intraventricular septum (IVS) and left ventricular free wall (LVFW) are located between angles $\theta$ varying from 0 to $0.3 \pi$ and from $0.7 \pi$ to $\pi$, respectively. The apex is located between IVS and LVFW. LVFW is divided at specific points $\theta=$ $[0.5 \pi, 0.6 \pi, 0.7 \pi, 0.8 \pi, 0.9 \pi, \pi]$.

Using coordinate system (1) we generated a finite difference (FD) mesh with constant $\Delta a(0.01 \mathrm{~cm})$ and $\Delta \theta$ $(0.01 \pi)$ mesh intervals, which spanned from 2 to $3 \mathrm{~cm}$ and from 0 to $\pi$ radians, respectively.

\subsection{Reaction Diffusion Model and Equations}

Simulations were done using the Bueno-OrovioFenton-Cherry reaction diffusion model (BOFC) [7]. The main part of the RD BOFC model which includes isotropic diffusion and the Laplacian for the spatial coordinates described in the Eq. (1) is defined by Eqs. (2)

$$
\begin{gathered}
\frac{\partial u}{\partial t}=D\left(\left(\cos ^{2} \theta+\frac{\sin ^{2} \theta}{s^{2}}\right) \frac{\partial^{2} u}{\partial a^{2}}+\right. \\
\left(-\sin 2 \theta \frac{\left(s^{2}-1\right)}{a s^{2}}\right)\left(\frac{\partial^{2} u}{\partial a \partial \theta}-\frac{1}{a} \frac{\partial u}{\partial \theta}\right)+ \\
\left.\left(\frac{\cos ^{2} \theta+s^{2} \sin ^{2} \theta}{(a s)^{2}}\right)\left(\frac{\partial^{2} u}{\partial \theta^{2}}-a \frac{\partial u}{\partial a}\right)\right)- \\
\sum_{i=f o, s o, s i} J_{i}(v, w, s)+J_{\text {Sim }}
\end{gathered}
$$

Here $u$ is a dimensionless variable which denotes a transmembrane potential. The magnitude of diffusion was set to $D=4.7 \mathrm{~cm}^{2} / \mathrm{sec}$. Values of $J_{i}$ represent the membrane ionic currents [7]. $J_{\text {stim }}$ indicates the stimulus current that excites the cells (see section 3.1). No flux Neumann boundary conditions were used in the direction normal to all the boundaries of the horseshoe geometry.

Myocyte cells APs include high depolarization and low repolarization rates. We reproduced this behavior in all different parts of the LV including epicardial, endocardial and mid-myocardial zones. BOFC parameters were set in the way to reflect that within epicardial and endocardial cells APs are normally shorter than in midmyocardial cells (Fig.3).

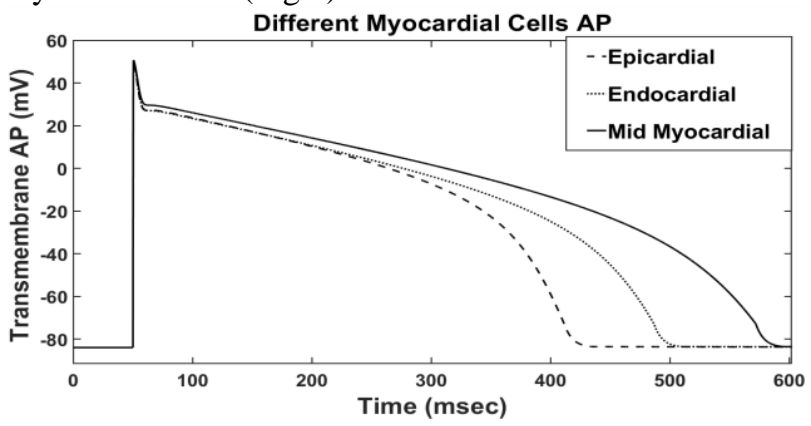

Figure 2. APs for different cell types. Dashed black line represents an epicardial cell AP, dotted gray line denotes an endocardial cell AP and solid black line represents a mid-myocardial cell AP.

\subsection{Simulating ECG}

Body surface ECG signals are calculated using Maxwell, Poisson, and continuity equations [2]. By assuming the thorax as an infinite homogeneous conductor, the in silico ECG can be calculated at the body surface electrode using Eq. (3)

$$
\operatorname{ECG}(t)=\iiint_{\tau} \frac{D \nabla V_{m} \cdot \vec{r}}{|r|^{3}} d \tau
$$

Where $D$ is the diffusive tensor, $V_{m}$ is the transmembrane potential, $r$ is the position between the electrode and the point where $V_{m}$ is measured in the medium, and $d \tau$ is the volume scaling factor. In our case the volume integral (3) was replaced with the area integral and was computed using trapezoidal method every $0.7 \mathrm{~ms}$.

The diffusive currents that wrap around the LV in a direction perpendicular to $\hat{e}_{a}$ and $\hat{e}_{\theta}$ (denoted as $\hat{e}_{\phi}$ ) are symmetric and also approximately perpendicular to a recording electrode (RE) placed outward from the apex in the direction approximately corresponding to precordial lead V4. Therefore, the $\hat{e}_{\phi}$ diffusive current is much less than diffusive currents in the directions $\hat{e}_{a}$ and $\hat{e}_{\theta}$.

\section{Results}

\subsection{Propagation in the $2 \mathrm{D}$ model}

The excitation which is initiated by a stimulus current $J_{\text {Stim }}$ raises the transmembrane potential above a threshold starting an AP sequence. We used a traveling $J_{\text {Stim }}$ that started at $\theta=0.25 \pi$ and ended at $\theta=0.9 \pi$ with the excitation range $a=2.05-2.17 \mathrm{~cm}$ and $\theta \pm 0.01 \pi$, respectively. Velocity of the $J_{\text {Stim }}$ excitation wave was equal to $4 \frac{\mathrm{m}}{\mathrm{s}}$.

All simulations were performed by implementing the second order spatial finite difference method (FDM) and the first order temporal FDM.
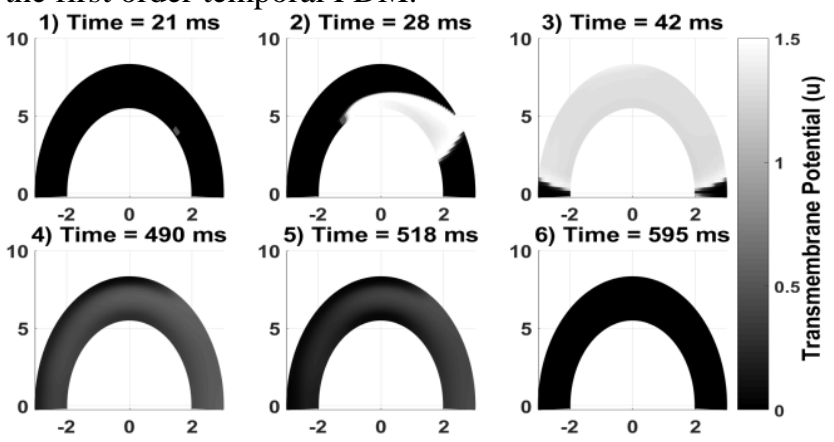
Figure 3. Propagation of the excitation within the 2D horseshoe model in Cartesian coordinates. Panel 1 shows $\mathrm{J}_{\text {Stim }}$ initial excitation of the medium at $\theta=0.25 \pi$ and $a=2.2$, which initiates the wave front shown by the light grey changing to black boundary. Panel 2 shows $J_{\text {Stim }}$ quickly depolarizing the $\mathrm{LV}$ at a constant $a=$ $2.2 \mathrm{~cm}$ and moving in the positive $\theta$ direction while the wave front continues to propagate at a slower speed outwards from where $J_{\text {Stim }}$ has originally excited the myocardium. Panel 3 shows $J_{\text {Stim }}$ stopping at $\theta=0.9 \pi$. Panel 4 shows the wave back starting in the epicardium before the endocardium and mid-myocardium; wave back is denoted as the area in transition from the black to the grey region. Panel 5 shows 2 wave backs propagating from endocardium and epicardium towards the midmyocardium. Panel 6 shows the entire medium returning to resting potential.

\subsection{Normal ECG}

Using the baseline parameters mentioned in 2.2, the Eqs. (2),(3) were solved under normal non-ischemic conditions. The RE was placed at $r=(0,10,0)$ and the evolution of excitation was like that shown in Fig.3. A typical normal body surface ECG signal is depicted in Fig. 4.

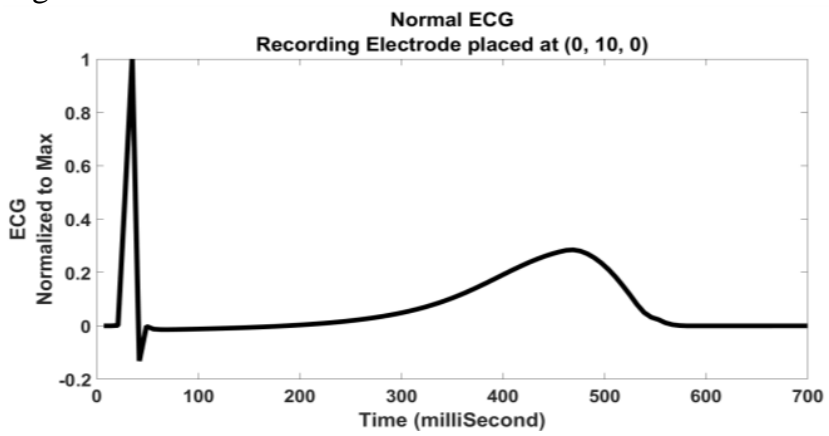

Figure 4. Normal ECG from the $2 \mathrm{D}$ model in Fig. 1. The $\mathrm{RE}$ is placed at $r=(0,10,0)$.

The key feature of the normal left ventricle ECG is characterized by a tall and narrow QRS complex and a relatively low amplitude and broad $\mathrm{T}$-wave. The $\mathrm{Q}$ wave in Fig. 4 is absent since only the left ventricle is being considered.

\subsection{Transmural myocardial infarction (MI)}

We modeled ischemic myocardium by elevating the parameter $u_{o}$, which controls the cellular resting potential (RP), in a certain area of myocardium. Typically ischemia starts in the endocardium and expands outwards rapidly towards the epicardium [8]. To represent this growth at different regions of our horseshoe model, the width was centered and varied in the range of $\theta=[0.5 \pi, 0.6 \pi, 0.7 \pi, 0.8 \pi, 0.9 \pi, \pi] \pm 0.05 \pi$ radians. The ischemic area for all $\theta$ increased in the $\hat{e}_{a}$ direction from $a=2$ (endocardium) to $a=3$ (epicardium). The ischemic ECG signals were computed for the different angles within the range indicated above.

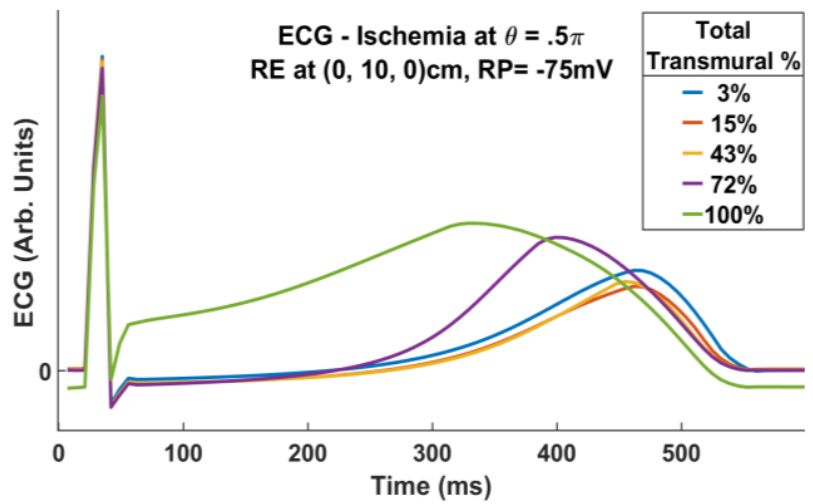

Figure 5. ECGs computed by increasing the ischemic area at $\theta=0.5 \pi$. Blue curve $(3 \%)$ is practically a normal ECG (Fig.4) and green curve represents a transmural MI.

Similar simulations were done at the other angular positions described above. The value of $u_{o}$ was increased to $.1(-75 \mathrm{mV})$ in ischemic area for all simulations. We observed that the ratio between ST deviation and T-wave amplitude $(\gamma)$ did not changed significantly with ischemic area for ischemic center locations confined at the higher angles $\theta$ greater than $0.7 \pi$. On the contrary, at $\theta<0.7 \pi$ we noted a sharp increase in $\gamma$ for ischemic areas greater than $\sim 1.4 \mathrm{~cm}^{2}$.

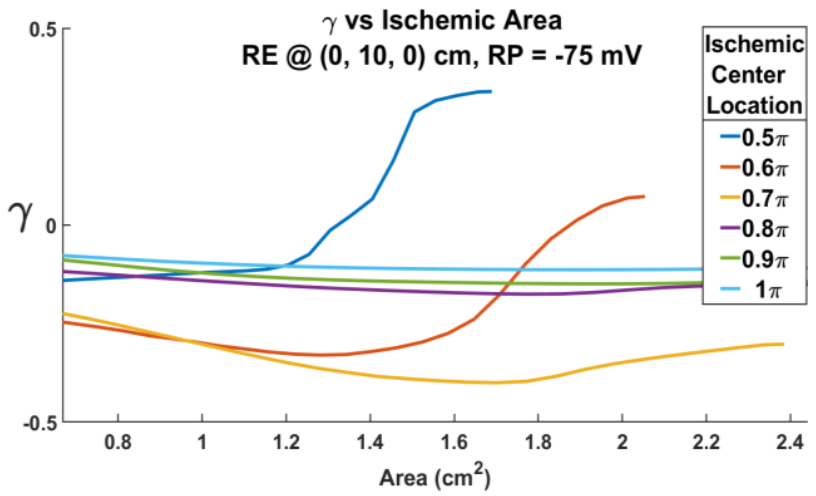

Figure 6. Dependence of $\gamma$ on the magnitude of ischemic area. Curves represent endocardium to epicardium growth at different locations in the LV apex and the LVFW. Legends show locations of the ischemic area center position. ST deviation was calculated as the ECG amplitude at the $\mathbf{J}_{60}$ point. The end of every curve designates the states of fully developed transmural ischemia designated as MI. 


\subsection{Calculated Severity of Ischemia}

Severity of ischemia was defined as the rate of changes in ischemic area per unit time (Eq.7).

$$
\text { Severity } \equiv \frac{\partial \text { Area }}{\partial t}=\frac{\partial \text { Area }}{\partial \gamma} \frac{\partial \gamma}{\partial t}
$$

The rate of changes in $\gamma$ per unit ischemic area can be recalculated from data shown in Fig. 6.

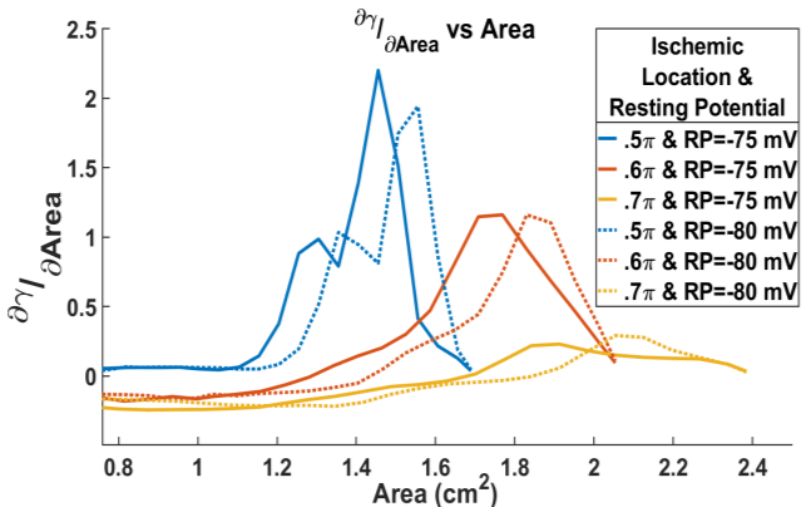

Figure 7. Changes in $\gamma$ per unit ischemic area are depicted for different ischemic growth at different angles $\theta$. The signals recorded at $\theta=0.5 \pi$ and $0.6 \pi$ demonstrate sharp changes like those shown in Fig. 6.

A coefficient $\left(\frac{\partial \gamma}{\partial \text { Area }}\right)^{-1}$ (see Fig. 7) can be considered in conjunction with another coefficient $\frac{\partial \gamma}{\partial t}$ determined from the original $\mathrm{V}_{4}$-type ECG signal. Combined these coefficients can be used for the estimates of severity of ischemia. Specifically, the average half maximum value of the $.5 \pi$ curves in Fig. $7(A=$ $1.04 \mathrm{~cm}^{-2}$ ) is a sufficiently accurate threshold to designate the fastest, and consequently the most dangerous ischemic growth.

\section{Discussion}

We demonstrated that it is possible to quantify the rate of ischemic growth using a single precordial lead aligned with the $\hat{e}_{\theta}$ direction in a 2D model of myocardium. Using $(A)^{-1}$ instead of the $\frac{\partial \text { Area }}{\partial \gamma}$ in Eq. 7 one can calculate a critical value of $\frac{\partial \gamma}{\partial t}$. For instance, if the thickness of the myocardium is $1 \mathrm{~cm}$ and ischemic area extends in the $\hat{e}_{\theta}$ direction by $2 \mathrm{~cm}$ then the value of $\frac{\partial \gamma}{\partial \mathrm{t}}$ may become greater than $0.018 \mathrm{~min}^{-1}$ indicating a quick spread of ischemia typical for the MI like ischemic event.

Finally, our model can be used in conjunction with machine learning to better understand more complex ischemic events.

\section{Conclusions}

Overall, we implemented a 2D reaction diffusion model to simulate the propagation of electrical excitation in the ischemic myocardium. It has been determined that the growth of the ischemic area results in increasing of the ratio $\gamma$. We found that the maximum $\frac{\partial \gamma}{\partial \text { Area }}$ value of $2.25 \mathrm{~cm}^{-2}$ indicates the strongest progression of the ischemic area. The critical (alert) value can be used to predict severe MI like ischemic growth if the value of $\frac{\partial \gamma}{\partial \mathrm{t}}$ is greater than $0.183 \mathrm{~min}^{-1}$.

\section{Acknowledgements}

This research was supported by the Joint School of Nanoscience and Nanoengineering. We would also like to thank Matthew Carnaghi for fruitful discussions.

\section{References}

[1] "Cardiovascular diseases (CVDs)." [Online]. Available: https://www.who.int/news-room/factsheets/detail/cardiovascular-diseases-(cvds). [Accessed: 06-Apr-2020].

[2] R. Plonsey and R. C. Barr, Bioelectricity: a quantitative approach. Springer US, 2007.

[3] A. Garny, D. Noble, and P. Kohl, "Dimensionality in cardiac modelling," Progress in Biophysics and Molecular Biology, vol. 87, no. 1 SPEC. ISS. Elsevier Ltd, pp. 47-66, 01-Jan-2005.

[4] C. Zeile, E. Scholz, and S. Sager, "A simplified 2D heart model of the Wolff-Parkinson-White Syndrome," IFAC-PapersOnLine, vol. 49, no. 26, pp. 26-31, Jan. 2016.

[5] R. E. Klabunde, "Cardiac electrophysiology: normal and ischemic ionic currents and the ECG," $A d v$. Physiol. Educ., vol. 41, no. 1, pp. 29-37, Mar. 2017.

[6] R. M. Shaw and Y. Rudy, "Electrophysiologic effects of acute myocardial ischemia: a theoretical study of altered cell excitability and action potential duration," Cardiovasc. Res., vol. 35, no. 2, pp. 256-272, Aug. 1997.

[7] A. Bueno-Orovio, E. M. Cherry, and F. H. Fenton, "Minimal model for human ventricular action potentials in tissue," J. Theor. Biol., vol. 253, no. 3, pp. 544-560, Aug. 2008.

[8] K. A. Reimer, J. E. Lowe, M. M. Rasmussen, and R. B. Jennings, "The wavefront phenomenon of ischemic cell death. 1. Myocardial infarct size vs duration of coronary occlusion in dogs," Circulation, vol. 56, no. 5, pp. 786-794, 1977.

Address for correspondence:

Loeffler, S E

JSNN, 2907 E. Gate City Blvd., Greensboro, NC 27401 seloeffl@uncg.edu 